\title{
Mussel Inspired Dynamic Cross-Linking of Self-Healing Peptide Nanofiber Network
}

\author{
Hakan Ceylan, Mustafa Urel, Turan S. Erkal, Ayse B. Tekinay, Aykutlu Dana,* \\ and Mustafa O. Guler*
}

\begin{abstract}
A general drawback of supramolecular peptide networks is their weak mechanical properties. In order to overcome a similar challenge, mussels have adapted to a $\mathrm{pH}$-dependent iron complexation strategy for adhesion and curing. This strategy also provides successful stiffening and self-healing properties. The present study is inspired by the mussel curing strategy to establish iron cross-link points in self-assembled peptide networks. The impact of peptide-iron complexation on the morphology and secondary structure of the supramolecular nanofibers is characterized by scanning electron microscopy, circular dichroism and Fourier transform infrared spectroscopy. Mechanical properties of the cross-linked network are probed by small angle oscillatory rheology and nanoindentation by atomic force microscopy. It is shown that iron complexation has no influence on self-assembly and $\beta$-sheet-driven elongation of the nanofibers. On the other hand, the organic-inorganic hybrid network of iron cross-linked nanofibers demonstrates strong mechanical properties comparable to that of covalently cross-linked network. Strikingly, iron cross-linking does not inhibit intrinsic reversibility of supramolecular peptide polymers into disassembled building blocks and the self-healing ability upon high shear load. The strategy described here could be extended to improve mechanical properties of a wide range of supramolecular polymer networks.
\end{abstract}

\section{Introduction}

Supramolecular polymers have become an attractive class of soft materials because their self-assembly is stimuli-responsive and reversible, and they possess self-healing properties. ${ }^{[1-5]}$ An unrivaled advantage of supramolecular polymer networks compared to traditional polymers is that many small-size building blocks could be synthesized with well-defined chemistry and organized into a particular architecture through noncovalent linkages. ${ }^{[5-7]}$ Due to their chemical versatility, short peptide sequences have emerged as one of the most referred building blocks within the context of supramolecular polymers. ${ }^{[4,5]}$ Although they are

H. Ceylan, M. Urel, T. S. Erkal, Dr. A. B. Tekinay,

Prof. A. Dana, Prof. M. O. Guler

Institute of Materials Science and Nanotechnology

National Nanotechnology Research Center (UNAM)

Bilkent University

Ankara 06800, Turkey

E-mail: aykutlu@unam.bilkent.edu.tr

moguler@unam.bilkent.edu.tr

DOI: 10.1002/adfm.201202291 largely designed to be utilized in tissue engineering and drug delivery, potential use of peptide based supramolecular polymers have extended into mechanical, electronic, and optical applications..$^{[5,8]}$

Despite the chemical and biological utilities of self-assembled peptide polymers, weak mechanical properties and limited control over these properties constitute a concern regarding their suitability in applications of a wider scope. ${ }^{[9,10]}$ To improve and tune mechanical properties, several independent strategies have been explored. An emerging approach is to form reversible cross-link points between supramolecular polymer chains, thereby keeping original advantages of the noncovalent assembly. Previously, Aulisa et al. explored contribution of dynamic $\mathrm{Mg}^{2+}$ and $\mathrm{PO}_{4}{ }^{3-}$ linkers on bulk elastic modulus of peptide hydrogels with various peptide sequences, compared to covalently cross-linked hydrogel control.[11] However, storage moduli of the physical hydrogels $(\approx 250 \mathrm{~Pa}$ on average) remained an order of magnitude lower than storage moduli of the covalently cross-linked system $(\approx 6000 \mathrm{~Pa})$. Using a similar approach, Stendahl et al. tuned $\mathrm{Ca}^{2+}$ ion concentration for gelation and modulation of mechanical properties of peptide amphiphile gels through interfiber cross-linking. ${ }^{[12]}$ This strategy enabled controlling storage modulus over three orders of magnitude. Nonetheless, using $\mathrm{Ca}^{2+}$ ions as both gelator and cross-linker brought additional issues regarding the degree of self-assembly. Since lowering $\mathrm{Ca}^{2+}$ concentration is not sufficient to screen all charges at neutral $\mathrm{pH}$, a significant portion of the peptide building blocks could not participate in nanofiber formation. Therefore, a main drawback of mechanical tunability in this system is that the elastic modulus was strictly coupled to the degree of selfassembly. In another strategy, Paramonov et al., and Pashuck et al. proposed that manipulation of peptide sequence dictating the secondary structure could provide control over bulk viscoelastic properties. ${ }^{[13,14]}$ Although some remarkable conclusions were drawn regarding the impacts of amphiphilic packing and orientation of building blocks on bulk elasticity, tunability range remained less than an order of magnitude. Taken altogether, alternative approaches for improving and controlling mechanical properties of supramolecular peptide networks, 


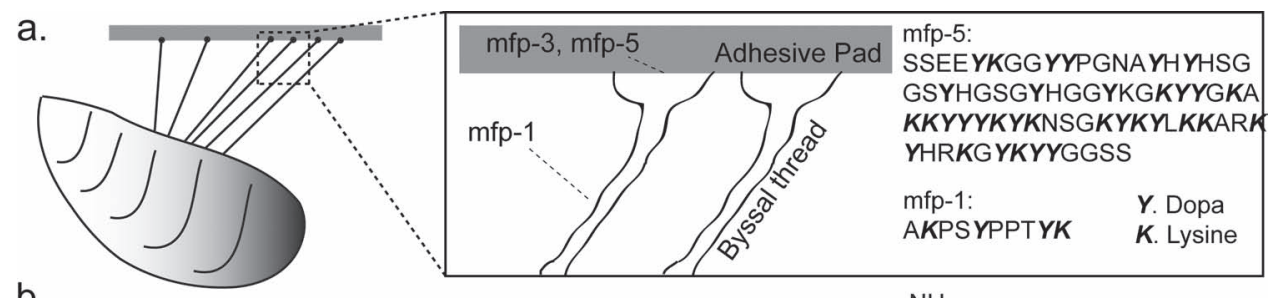

b.<smiles>CCCCCCCCCCCC(=O)NC(C(=O)NC(C(=O)NC(C)C(=O)NCC(=O)NC(CCCCCN)C(=O)N[C@@H](Cc1ccc(O)c(O)c1)C(N)=O)C(C)C)C(C)C</smiles><smiles>CCCCCCCCCCCCC(=O)NC(C(=O)NC(C(=O)NC(C)C(=O)NCC(=O)N[C@@H](CCCCN)[C@H](N)C(N)=O)C(C)C)C(C)C</smiles>

c.

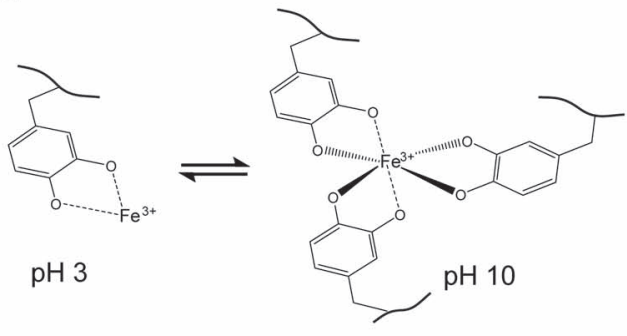

Iron-mediated reversible coordination

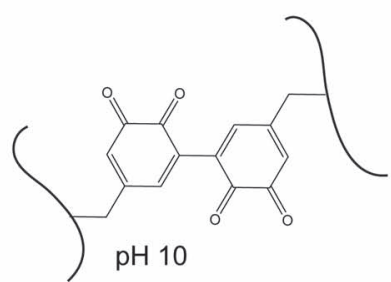

$\mathrm{pH}$-triggered irreversible curing

Figure 1. Mussel-inspired mechanical enhancement strategy for supramolecular peptide network. a) Schematic of a marine mussel affixing to a surface. Dopa and lysine are the two key residues in mussel adhesive proteins (here only mfp-1, mfp-3, and mfp-5 are shown) for mussel adhesion and curing. ${ }^{[41,42]}$ b) Chemical representation of DopaK-PA and K-PA building blocks of supramolecular peptide networks introduced in this study. c) In the presence of iron, tris $\mathrm{Fe}(\mathrm{Dopa})_{3}$ complexes form dynamic cross-link points in mussel adhesive proteins of the byssus while basic $\mathrm{pH}$ triggers oxidation mediated covalent cross-linking of the proteins.

while retaining intrinsic reversibility and self-healing ability, are required.

Marine organisms have unique properties that enable them to survive the destructive conditions of ocean. These characteristics provide a plethora of inspiration to surmount challenges for development of advanced functional materials. A remarkable example is the adaptation of common blue mussel, Mytulis edulis, to remain sessile, i.e., nonmotile, under the highly unstable conditions of intertidal zones where irregularities in salinity, ceaseless wearing of the ocean waves, and sharp fluctuations of temperature and $\mathrm{pH}$ create an environment of harsh extremes. In order to overcome these, mussels produce a special adhesive containing hierarchically organized mussel adhesive proteins with a high content of 3,4-dihydroxyL-phenylalanine (Dopa) residues (Figure 1a). Catecholic units of Dopa are regarded as vital for adhesion onto a wide range of organic and inorganic substrates and for cross-linking reactions of the cohesive curing. ${ }^{[15-19]}$ Because of the simplicity of conjugation of Dopa molecule onto synthetic materials and versatility of the substrates it could bind to, mussel mimetic adhesion has become an established strategy for developing biomimetic adhesion systems. ${ }^{[15,16,20-22]}$ In contrast, there are only a few examples that recapitulate the chemistry of mussel cuticle in synthetic materials towards materials science applications. ${ }^{[17,23,24]}$ Ex vivo studies showed that mussel adhesive proteins could be cross-linked through metal-ion-complexation or oxidation mediated covalent reactions (Figure 1c). ${ }^{[25]}$ At alkaline $\mathrm{pH}$, Dopa is easily oxidized to highly reactive quinone and semiquinone species that further react with each other to form covalent cross-link points. ${ }^{[16,26]}$ However, the main organization of mussel cuticle is formed by coordination complexes between Dopa and metal ions, predominantly by ferric iron ions. Under basic conditions $(\mathrm{pH} \approx 8.5)$, Dopa and iron ions form bis $\mathrm{Fe}(\mathrm{Dopa})_{2}$ and tris $\mathrm{Fe}(\mathrm{Dopa})_{3}$ complexes. Shafiq et al. reported that conjugation of a nitro group to dopamine could reduce $\mathrm{pKa}$ of the catechol hydroxyl groups to $\approx 6.5$, revealing that iron mediated cross-linking could be controlled through chemical modifications on the catechol and hence widening the scope of utility of this material. ${ }^{[2]]}$ While reversible, Dopa-iron bis- and tris-complexes have one of the highest known stability 
constants $\left(\log K_{s} \approx 37-40\right)$ of metal-ligand chelates and crosslinks provide the cuticle both hardness and self-healing ability after fracture. ${ }^{[27,28]}$ This unique strategy has inspired us to apply metal-ligand coordination as a mechanical reinforcing strategy for self-assembled peptide networks.

Herein, we show that reversible cross-linking of self-assembled peptide network with iron is a promising method to improve mechanical properties while retaining intrinsic selfhealing properties. For this purpose, we designed a musselinspired peptide amphiphile, lauryl-Val-Val-Ala-Gly-Lys-DopaAm (DopaK-PA) (Figure 1b). Similar to mussel adhesive proteins, self-assembled DopaK-PA network can be cross-linked either with iron incorporation or oxidative pathway. As a control of chemical cross-linking, we synthesized another mussel-inspired peptide amphiphile, lauryl-Val-Val-Ala-GlyLys-Am (K-PA). K-PA has the same sequence of DopaK-PA; however, it lacks Dopa (Figure 1b). As it takes place in mussels, $\mathrm{pH}$ dependent complexation of iron ions enabled formation of tris $\mathrm{Fe}(\mathrm{Dopa})_{3}$ complexes in DopaK-PA network without destructing the supramolecular order. In the absence of iron, catecholic units in the self-assembled network underwent oxidation followed by covalently cross-linking of nanofibers. Since K-PA lacked Dopa, its nanofibers were physically entangled and hence demonstrated weak mechanical properties. We revealed that the mechanical properties of the iron cross-linked DopaKPA network matched the properties of covalently cross-linked DopaK-PA network. Strikingly, iron cross-linking had a dynamic nature; it retained its $\mathrm{pH}$ dependent reversibility and demonstrated self-healing properties similar to uncross-linked K-PA network. On the other hand, covalent cross-linking inhibited $\mathrm{pH}$ response and self-healing properties of the self-assembled DopaK-PA network. Both cross-linking strategies were entirely orthogonal to the self-assembly mechanism. These results highlighted the significance of metal coordination in a supramolecular network to improve mechanical properties without causing mineralization or interfering with the self-assembly mechanism. Because Dopa incorporation into synthetic molecules is relatively simple, this strategy can be extended into other systems operating under neutral or basic $\mathrm{pH}$.

\section{Results and Discussion}

\subsection{Self-Assembly of Mussel-Mimetic Peptide Building Blocks}

A peptide amphiphile molecule is composed of several functional modules carrying the necessary information to selfassemble into nanofibers and to manifest its desired chemical or biological functionality (Figure 1b). Our amphiphile design included a hydrophobic lauryl group attached to the N-terminus of the peptide segment to force packing the building blocks into micellar assemblies. In favor of entropic gain, the hydrophobic segment was buried into the nanofibers to expose hydrophilic peptide sequence to the aqueous environment. The lauryl group was attached to Val-Val-Ala-Gly peptide sequence, whose amide backbone facilitated the secondary structure through hydrogen bonds in the direction of nanofiber elongation. Lys residue was incorporated as a switch for the self-assembly. Supramolecular ordering of the peptide amphiphile molecules is robustly promoted upon neutralization where strong repelling forces of the same charged species are deactivated and hydrophobic interactions dominate. ${ }^{[29,30]}$ Therefore, deprotonation of positively charged side chains on both DopaK-PA and K-PA acted as a switch for the self-assembly into nanofibers. Further, Lys residue is known to play a distinct role in mussel adhesion and curing chemistry. Positively charged Lys residue is abundantly found in major mussel adhesive proteins, $\mathrm{mfp}-1, \mathrm{mfp}-3$, and mfp-5, imparting a cationic nature to the mussel adhesive proteins. ${ }^{[16,19,31]}$ Titration of DopaK-PA and K-PA solutions with $\mathrm{NaOH}$ revealed their isoelectric points to be 8.9 and 9.9, respectively, which are very close to the pIs of $\mathrm{mfp}-1$ ( $\mathrm{pH} \approx 10$ ), mfp-3 $(\mathrm{pH} \approx 8-10)$, and $\mathrm{mfp}-5$ ( $\mathrm{pH} \approx 9-10)$ (Supporting Information Figure S2). ${ }^{[19]}$ Even though its particular role is still unknown, recent attempts to imitate mussel adhesion mechanism in synthetic materials have focused on utilizing Dopa and Lys residues together. ${ }^{[15,16,20,31]}$ It is currently considered that the excess positive charge forms columbic interactions with surfaces that mussels adhere in their native environment, such as rocks that are highly rich in negatively charged silicates and aluminates. ${ }^{[31]}$ In fact, Dopa and Lys are utilized not only in mussel adhesives but also in natural adhesives of other organisms including sandcastle worm, Phragmatopoma californica. ${ }^{[18]}$ The commonality of this system indicates that an exclusive interaction and/ or cooperation between Dopa and Lys may have provided a universal solution for adhesion of marine animals.

$\mathrm{Fe}^{3+}$ has low solubility at neutral or basic $\mathrm{pH}$ at room temperature as it readily precipitates in hydroxylated form. In order to form iron cross-linked peptide gels, $\mathrm{FeCl}_{3}$ solution was initially mixed with DopaK-PA solution at $\mathrm{pH} \approx 3$ with a final stoichiometric ratio of 3:1 [Dopa:Fe]. Within seconds after mixing, the color of the mixture turned to dark green indicating formation of mono Fe(Dopa) complex (Supporting Information Figure S3). ${ }^{[17,26]}$ Incorporation of ferric ions by themselves did not induce self-assembly, as determined from circular dichroism spectrum, and the mixture remained dissolved in the solution (Supporting Information Figure S4). To induce selfassembly, $\mathrm{pH}$ of the solution was increased to $\approx 10$ (to deprotonate $\varepsilon$-amine of lysine residue) by adding $\mathrm{NaOH}$. Immediate color change from dark green to wine red accompanied the self-assembly process. Color change indicated a transition from mono $\mathrm{Fe}(\mathrm{Dopa})$ complex to tris $\mathrm{Fe}(\mathrm{Dopa})_{3}$ complex. ${ }^{[17,26]}$ The $\mathrm{pH}$ dependent absorbance shifts were identical to the color changes of catechol- $\mathrm{Fe}^{3+}$ coordination status reported previously (Supporting Information Figure S5). ${ }^{[26]}$ This strategy is analogous to mussels that integrate $\mathrm{Fe}^{3+}$ into densely cross-linked granules inside the cuticle layer of byssal threads. ${ }^{[27,28]}$ Inside acidic $(\mathrm{pH} \approx 5)$ intracellular granules of byssal gland cells, a proteinaceous precursor of glue cocktail is produced. At acidic $\mathrm{pH}$, catechol units of Dopa are not oxidized spontaneously and coordinates with $\mathrm{Fe}^{3+}$ as a mono complex. Once released into the ocean, the alkaline environment $(\mathrm{pH} \approx 8.5)$ directs bis- and tris-Fe(Dopa) ${ }_{3}$ complexation. ${ }^{[17]}$ In our system, since the initial mono complexation of $\mathrm{Fe}^{3+}$ to DopaK-PA took place in the solution phase (at acidic $\mathrm{pH}$ ) homogeneously, $\mathrm{Fe}(\mathrm{Dopa})_{3}$ cross-link points were dispersed highly uniformly inside the gel after $\mathrm{pH}$ increase. In the absence of iron, DopaK-PA followed a totally different reaction pathway at $\mathrm{pH} \approx 10$ (Supporting Information 

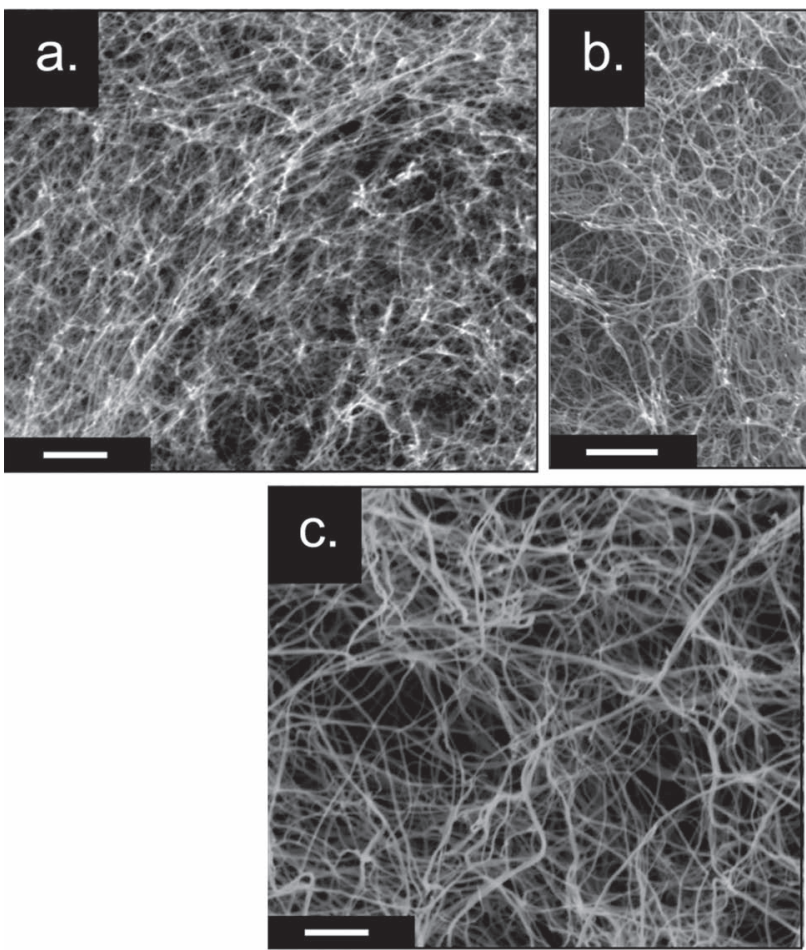

Figure 2. SEM images of the mussel inspired, self-assembled peptide nanofibers. a) Iron crosslinked DopaK-PA/Fe(III) network. b) Covalently cross-linked DopaK-PA network. c) Physically entangled nanofibers of K-PA network. Scale bar: $500 \mathrm{~nm}$.

Figure S3 and Figure S5). ${ }^{[26]}$ Catechol units of Dopa are not stable at basic $\mathrm{pH}$ and are rapidly oxidized to quinone and semiquinone, which further react with each other to form covalent linkages. ${ }^{[16,26]}$ Addition of $\mathrm{NaOH}$ to DopaK-PA at $\mathrm{pH} \approx 3$ caused a color change to yellow that gradually developed into pale yellow, indicating oxidation-driven covalent cross-linking of the network (Supporting Information Figure S3). Because basic $\mathrm{pH}$ is required for both self-assembly of the building blocks and cross-linking of the network (either iron-coordinated or covalent cross-linking), there was a competition between the two reactions that occur concomitantly. However, SEM images show that the supramolecular order of the nanofibrous networks were preserved in both cross-linking schemes, indicating that self-assembly had a faster rate of reaction (Figure 2a,b). Likewise, self-assembly of K-PA was induced at $\mathrm{pH} 10$ resulting in a nanofibrous network (Figure 2c). The network of K-PA was held intact through weak noncovalent interfiber interactions, such as van der Waals, dipoledipole, hydrogen bonding, and columbic interactions.

\subsection{Characterization of the Secondary Structure of the Peptide Nanofibers}

In order to probe the secondary structure of peptide nanofibers, circular dichroism (CD) and FT-IR spectroscopy were employed.

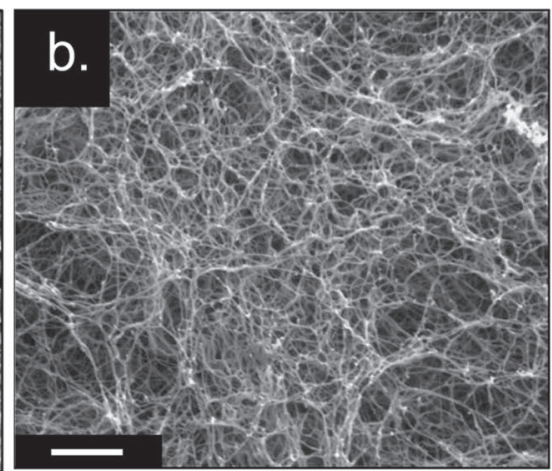

Circular dichroism spectra revealed a maximum at $203 \mathrm{~nm}$ and minimum at $220 \mathrm{~nm}$, which shows that the predominant organization of building blocks at $\mathrm{pH} \approx 10$ was $\beta$-sheet in all three groups (Figure 3a). ${ }^{[11]}$ In FT-IR analysis, amide I vibration mainly originates from the carbonyl stretching aligned with hydrogen bonding direction in the backbone of polypeptides; and therefore contains information regarding the secondary structure. In all three peptide nanofibers, amide I peaks were located between $1630-1640 \mathrm{~cm}^{-1}$, revealing $\beta$-sheet organization as shown in CD analysis (Figure 3b). ${ }^{[14]}$ These results show that chemical cross-linking inside the network did not change the supramolecular organization of the constituent building blocks of the nanofibers. Thus, mussel inspired protocol for iron cross-linking is a safe method to form interfiber cross-links and can be applied to similar self-assembly-based structures without harming the supramolecular order.

\subsection{Bulk Rheological Analyses of the Cross-Linked Supramolecular Network}

Gelation kinetics and viscoelastic properties at equilibrium are critical material properties for a gel, which dictates its suitability for the desired use. ${ }^{[32]}$ Gelation kinetics was monitored through time-sweep analysis in linear viscoelastic range. In rheological terms, gelation occurs at a time point at which the storage modulus, i.e., energy stored during deformation, exceeds loss modulus, i.e., energy dissipated during deformation. Within $1 \mathrm{~h}$, the storage and loss moduli of all three groups almost reached plateau (Figure $4 \mathrm{a}$ and Supporting Information Figure S6a). Therefore, the rest of the rheological tests were carried out after $1 \mathrm{~h}$ equilibration period. The storage modulus of iron cross-linked network (DopaK-PA/Fe(III)) was greater than storage modulus of covalently cross-linked DopaK-PA network during the first $30 \mathrm{~min}$, after which there was no significant difference between them. This indicates iron cross-linking takes place at a faster a.

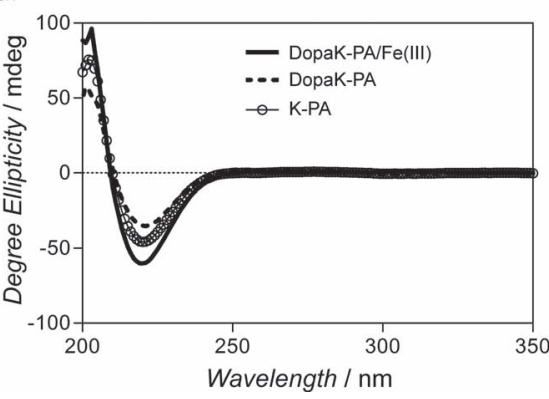

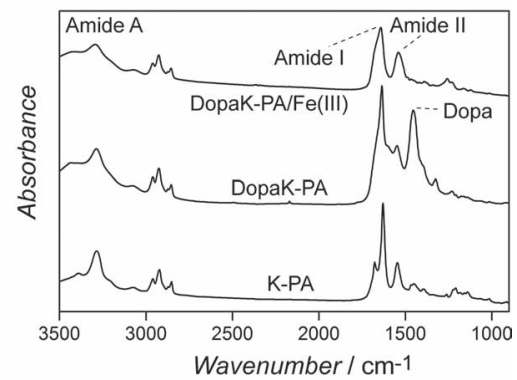

Figure 3. Secondary structure analyses of the mussel-inspired peptide nanofibers. a) Circular dichroism, b) FTIR spectra at $\mathrm{pH} \approx 10$. 
a.
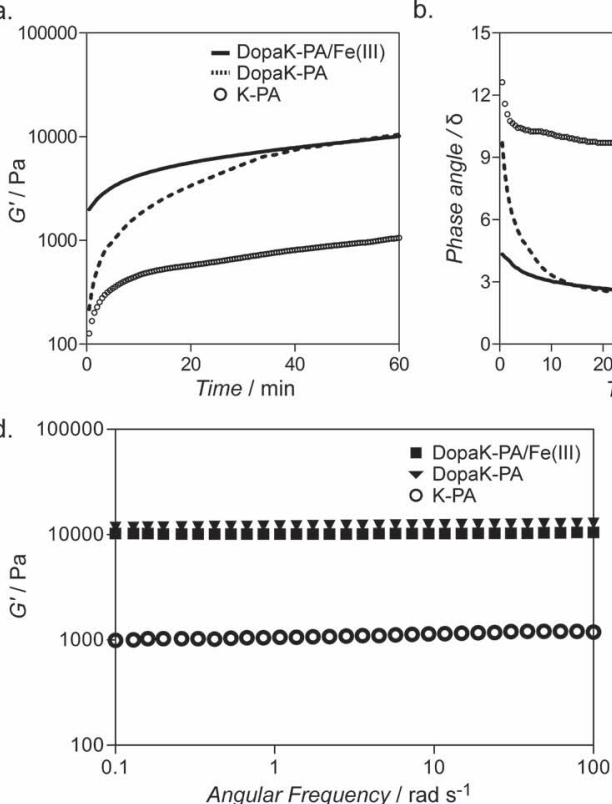

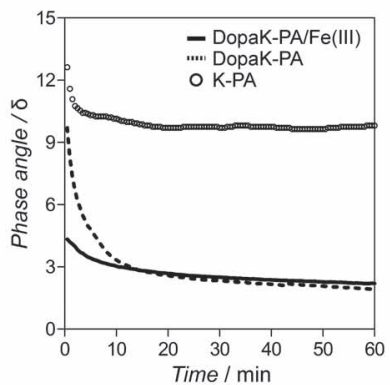

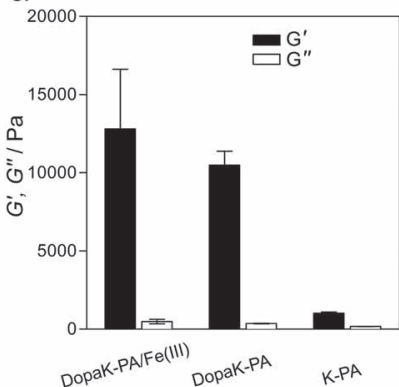

e.

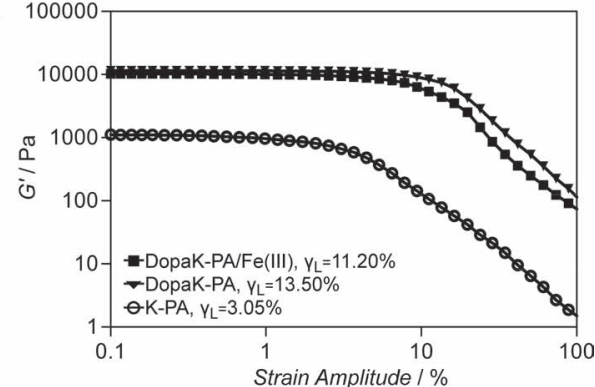

Figure 4. Rheological characterizations of mussel inspired peptide gels at 1 wt\% concentration. a) Gelation kinetics, b) phase angle as a function of time, c) Equilibrium moduli at $1 \mathrm{~h}$, d) frequency sweep test, and e) amplitude sweep test.

rate than covalent cross-linking whilst the storage moduli are comparable at equilibrium. A more elaborative way to interpret kinetics of gelation and to elucidate the impact of crosslinking on the mechanical properties of networks is to convey the phase angle as a function of time. ${ }^{[33]}$ The storage $\left(G^{\prime}\right)$ and loss $\left(G^{\prime \prime}\right)$ moduli are related by $\tan (\delta)=G^{\prime \prime} / G^{\prime}$, where $\delta$ is the phase angle and $\tan (\delta)$ is the loss (damping) factor. Gelation takes place if $\delta$ falls below $90^{\circ}$, or $G^{\prime \prime} / G^{\prime}<1$. In other words, as $\delta$ approaches from $90^{\circ}$ to $0^{\circ}$, the network gains an elastic character and loses its viscous character, and vice versa. From $t_{0}$ to $t_{1 \mathrm{~h}}, \delta$ remained lower than $90^{\circ}$ with a logarithmical decrease over time in all three networks (Figure 4b). Although rheological test was started immediately upon $\mathrm{pH}$ increase to $\approx 10$, we were not able to catch the sol-gel transition point (where $\delta$ was greater than $90^{\circ}$ ), as the rate of self-assembly was exceedingly high (probably in the time scale around or lower than milliseconds). Regarding gelation kinetics, K-PA gel reached plateau at a faster rate than both DopaK-PA/Fe(III) and DopaK-PA (Figure 4b). The value $\delta$ of K-PA almost reached plateau within as fast as $3 \mathrm{~min}$ while it took $8 \mathrm{~min}$ for DopaK-PA/Fe(III) and 11 min for DopaK-PA revealing that self-assembly was a faster process than cross-linking. This explains the preservation of supramolecular architecture upon cross-linking, which concomitantly took place with self-assembly at $\mathrm{pH} 10$. The faster reaction rate of iron coordination compared to oxidative crosslinking is a significant phenomenon for marine organisms as well, because formation of highly organized and dense granules of iron cross-links on the cuticle of mussel byssal threads needs to be competitive against the oxidation. Previous reports about peptide amphiphiles showed that physically entangled (noncovalent cross-linking) supramolecular peptide gels had a loss factor in the range of $0.20-0.10$ at equilibrium. ${ }^{[14,34]}$ This value was in agreement with the loss factor of K-PA (0.170)
(Supporting Information Figure S6b). As expected, chemical cross-linking caused a sharp $(\approx 5$-fold $)$ decrease of this value although the values were comparable for DopaK-PA/Fe(III) (0.038) and covalently cross-linked DopaK-PA network (0.036). Similarly, the phase angles of covalently cross-linked DopaK-PA, iron coordinated DopaK-PA/Fe(III), and K-PA networks at the end of $1 \mathrm{~h}$ were $1.96^{\circ} \pm 0.03^{\circ}, 2.22^{\circ} \pm 0.02^{\circ}$, and $9.78^{\circ} \pm 0.03^{\circ}$, respectively. This pronounced difference in the loss factor and $\delta$ between chemically cross-linked and physically cross-linked networks were because of the decrease in dissipated energy (loss modulus) and increase in stored energy (storage modulus). During cross-linking, shrinking mesh size causes some portion of water to be excluded from the gel (decreased average distances in interfiber interaction points), thereby diminishing the viscous character. SEM images revealed shrinking in average mesh size (Figure 2). As a result, more energy was stored compared to that dissipated. On the contrary, the increase in loss factor and phase angle was due to the relative increase in dissipated energy which was the result of partial breaks within and between the nanofibers in the network. Accordingly, iron coordination or covalent bonding could act as bridges to link such breaks inside the network.

After $1 \mathrm{~h}$ of equilibration, average bulk storage moduli $\left(G^{\prime}\right)$ of $1 \mathrm{wt} \%$ DopaK-PA/Fe(III) and DopaK-PA gels were found to be comparable $\left(1.28 \times 10^{4} \pm 3.81 \times 10^{3} \mathrm{~Pa}\right.$ and $1.05 \times 10^{4} \pm$ $0.91 \times 10^{3} \mathrm{~Pa}$, respectively) (Figure 4c). There was no statistical difference between these magnitudes. On the other hand, storage modulus of K-PA gel was less than an order of magnitude $\left((1.18 \pm 0.84) \times 10^{3} \mathrm{~Pa}\right)$ of either of the cross-linked gels $(p<0.05)$, signifying the impact of chemical cross-linking on bulk viscoelasticity of self-assembled peptide network. After $1 \mathrm{~h}$ of equilibration, storage moduli of all gels demonstrated a frequency-independent behavior and no crossover was observed 
at lower frequencies, indicating that gelation was completed and nanofibers were linked through dense interaction points (either physical interactions or chemical crosslinking) (Figure 4d). ${ }^{[35]}$ To investigate the relationship between storage modulus and strain amplitudes, we performed a amplitude sweep test. Beyond certain strain amplitude, called the limiting strain amplitude, or $\gamma_{L}$, the network showed a transition from linear to nonlinear viscoelastic behavior. Below $\gamma_{L}$, the storage modulus is independent of the strain amplitude and constitutes the linear viscoelastic range. Limiting strain amplitudes of the DopaK-PA/Fe(III), DopaK-PA, and K-PA were $11.20 \%, 13.50 \%$, and $3.05 \%$, respectively (Figure 4e). This difference in $\gamma_{\mathrm{L}}$ shows that compared to K-PA, DopaK-PA/Fe(III) could withstand more than three times higher shear strain, while, for DopaK-PA, plastic deformation occurred after approximately four times higher strain. In other words, chemical crosslinking inside the network imparted resistance to deformation until intrafiber interactions were broken at $\gamma_{\mathrm{L}}$. As initial monomer concentration increased, the difference of equilibrium storage moduli between chemically cross-linked DopaK-PA/Fe(III) and uncross-linked K-PA increased due to the increase in the total number of cross-link points inside the network and increased number of elastically active chains (Supporting Information Figure S7).

\subsection{Nanomechanical Characterizations of the Cross-Linked Nanofibers}

In order to gain further insight into the impact of cross-linking on mechanical properties of the mussel inspired peptide network, elasticity of the constituent nanofibers and nanofiber bundles were investigated using double-pass force-distance mapping through atomic force microscopy (AFM) nanoindentation. ${ }^{[36]}$ It was observed that DopaK-PA/Fe(III) and DopaKPA gels can withstand greater strains (ca. 11-13\%) than K-PA gels (ca. 3\%) before losing structural integrity and losing their elastic moduli (Figure 4e). Previously, increasing strength of inter and intra-fiber bonds were found to be related to improved stability and elastic modulus of peptide nanofiber gels. ${ }^{[34]}$ Through nanoindentation measurements, response to deformation can be observed by comparing elasticity and adhesion values extracted from approach (of the AFM tip) and retraction components of the force-distance curve. During approach and retraction of the AFM tip, elastic moduli of the iron crosslinked DopaK-PA/Fe(III) and covalently cross-linked DopaK-PA nanofibers exhibited values in the order of $10^{8} \mathrm{~Pa}$ (Figure 5a,b). In contrast, K-PA nanofibers exhibited a double peak, which we interpreted to be due to crushing of thinner fibers and apparent increased modulus caused by partial appearance of the hard (modulus > $100 \mathrm{GPa}$ ) silicon substrate. ${ }^{[37]}$ All measurements were performed with similar peak pressing forces on the order of $10 \mathrm{nN}$. In the retraction curves, DopaK-PA/Fe(III) and DopaK-PA nanofibers still exhibited well defined single peaks, while K-PA fibers displayed even better resolved doublepeaks (Figure 5b). The lower peak in the histogram of K-PA fibers displayed slightly smaller modulus than DopaK-PA/ Fe(III) and DopaK-PA nanofibers. These observations suggest that chemical cross-linking not only improved the mechanical properties of the network as a whole, but also strengthened its individual fibrous components. Dense interfiber cross-linking increases bundling of the individual fibers, which, requires greater force for deformation of thicker bundles. Elasticity measurements during approach of the AFM tip was regarded as mechanical properties of undisturbed nanofibers. On the other hand, pressing nanofibers with AFM tip could cause irreversible deformations (due to breaking of intrafiber and interfiber bonds), which influence their mechanical response during retraction of the tip. Before and after indentation with the AFM tip, adhesion histograms also shifted towards higher adhesion forces for all fibers (Figure 5c,d). Here, DopaK-PA/ $\mathrm{Fe}(\mathrm{III})$ and DopaK-PA displayed adhesion histograms with well defined single peaks, whereas K-PA displayed a wide distribution of adhesion forces, greatly increased as compared to the DopaK-PAs. The increase in the adhesion between the tip and the nanofiber is attributed to the presence of unsaturated bonds, which is greatest for K-PA nanofibers. This supports the hypothesis that DopaK-PA/Fe(III) and DopaK-PA nanofibers are composed of peptides making stronger and multiple bonds among themselves, while K-PA nanofibers possibly consist of peptides which make weaker bonds among themselves, and are therefore not in their minimal energy configuration. According to the nanoindentation results (Figure 5c,d), K-PA nanofibers have greater number of unsaturated bonding sites compared 
to DopaK-PA/Fe(III) and DopaK-PA fibers. A similar observation on the relation between observed adhesion, structural integrity and elastic modulus of nanofibers was reported for amyloid-like fibers. ${ }^{[38]}$

\subsection{Influence of Temperature on the Curing of Mussel Inspired Cross-Linking in the Supramolecular Networks}

In order to investigate the curing effect of temperature on mussel-inspired networks and the constituent nanofibers, the system was heated to $80{ }^{\circ} \mathrm{C}$ followed by cooling back down to room temperature (Supporting Information Figure $\mathrm{S} 8 \mathrm{a}-\mathrm{C}$ ). Noncovalent interactions of adjacent monomers within the nanofibers and between the fiber chains are sensitive to even small temperature changes and network tends to collapse during heating. ${ }^{[34]}$ Both iron cross-linked and covalently cross-linked networks first showed (up to $\approx 40{ }^{\circ} \mathrm{C}$ ) a tendency to break apart assessed by the collapsing storage modulus. Above this temperature and up to $80{ }^{\circ} \mathrm{C}$, the storage modulus suddenly increased linearly to recover the initial storage moduli. This indicated that heating provided a dynamic platform that caused formation of new cross-link points within the network. As cooling back to room temperature, storage moduli of both networks further increased up to three folds of the equilibrium moduli (kinetic equilibrium). During cooling, monomer packing into the nanofibers and nanofiber organization within the network allows more efficient organization culminating in higher network stiffness. Further heating and cooling both iron and covalently cross-linked networks followed the previous cooling path and the system reached to its thermodynamic equilibrium. ${ }^{[39]}$ AFM nanoindentation also verified enhanced stiffness of nanofibers and bundles after heating and cooling (Supporting Information Figure S8d,e). On the other hand, physically entangled K-PA network showed a consistent decrease during heating. Upon cooling, packing effect led to enhanced storage modulus at room temperature. Further heating and cooling followed a similar trend and the network did not demonstrate curing behavior.

\section{6. $\mathrm{pH}$ Dependent Reversibility of the Mussel Inspired Supramolecular Network}

Due to the ionic nature of the molecule, $\mathrm{pH}$ is an essential stimuli to trigger reversible assembly of peptide amphiphile molecules into supramolecular polymer networks. ${ }^{[29,30]}$ As disassembly of the network is strongly coupled with the viscoelastic behaviors of the networks, decrease in the storage modulus upon lowering $\mathrm{pH}$ into acidic zone is attributable to the degree of reversibility of the assembly. After $1 \mathrm{~h}$ of equilibration at $\mathrm{pH} \approx 10, \mathrm{pH}$ was decreased back to $\approx 3$ by addition of $\mathrm{HCl}$ solution. Physically entangled K-PA nanofibers rapidly disassembled at $\mathrm{pH} \approx 3$, with $\approx 90 \%$ decline in storage modulus within 10 min (Figure 6a). Similarly, DopaK-PA/Fe(III) network disorganized into mono Fe(Dopa) complex building blocks, as the color change from wine red into dark green indicated (Supporting Information Figure S3). Decrease in storage modulus of tris $\mathrm{Fe}(\mathrm{Dopa})_{3}$ cross-linked gel was $87 \%$, comparable to that of K-PA. This indicates that $\mathrm{Fe}(\mathrm{Dopa})_{3}$ complex is fully reversible into mono Fe(Dopa) complex with pH. DopaK-PA/Fe(III) gel lost all of its mechanical strength upon lowering $\mathrm{pH}$ while it rapidly recovered its mechanical properties after increasing $\mathrm{pH}$ (Figure 6b). A few past studies reported oxidation of catechol and reduction of $\mathrm{Fe}^{3+}$ because of their similar redox potential $(\approx 0.75 \mathrm{~V}) .{ }^{[40]}$ Based on the degree of decrease in mechanical properties of DopaK-PA/Fe(III) and colorimetric analysis, we did not notice iron mediated oxidation within the time scale of these experiments. In contrast, decrease in storage modulus in DopaK-PA was only $31.4 \%$ and no apparent color reversibility was observed, demonstrating that covalent cross-linking irreversibly locked the network. 


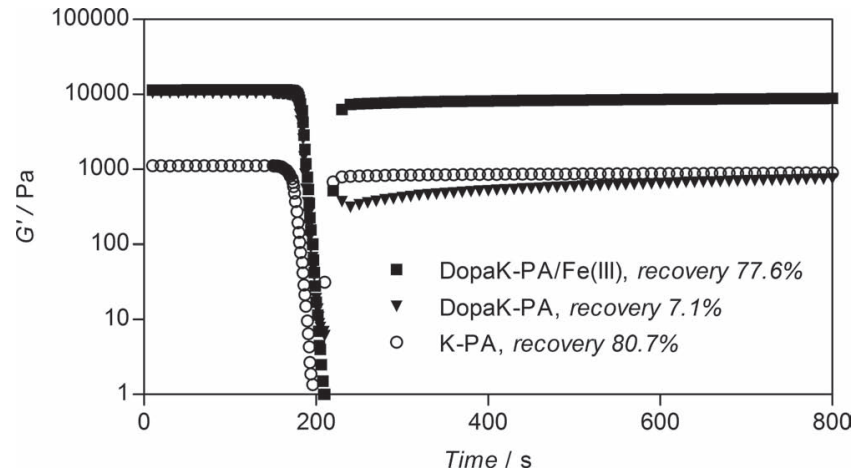

Figure 7. Self-healing of the mussel inspired peptide gels. Recovery after high shear load monitored via storage modulus as a function of time.

\subsection{Self-Healing Properties of the Networks}

To test the self-healing ability upon high shear load far beyond linear viscoelastic behavior (1000\%), we performed thixotropic test. Under such high deformation, both covalent and noncovalent bonds within and between the nanofibers are broken; therefore, noncovalent bonds are expected to recover rapidly after the load is removed. Within 10 min after load was applied, DopaK-PA/Fe(III) recovered $77.6 \%\left(8.85 \times 10^{3} \mathrm{~Pa}\right)$ of its original storage modulus at $1 \mathrm{~h}$ (Figure 7). Comparably, the recovery of K-PA was $80.7 \%\left(8.98 \times 10^{2} \mathrm{~Pa}\right)$. Following deformation at $1000 \%$ shear strain, noncovalent interactions that drive self-assembly of the peptide amphiphiles were mostly restored rapidly in both K-PA and iron cross-linked DopaK-PA/ $\mathrm{Fe}(\mathrm{III})$. Fe(III) ions diffused in and rebound to the network in a highly reversible fashion. In contrast, covalently cross-linked DopaK-PA recovered by only $7.1 \%\left(7.51 \times 10^{2} \mathrm{~Pa}\right)$, because covalent bonds inside the nanonetwork could not be recovered after structural deformation. This shows that DopaK-PA could withstand slightly larger strains before plastic deformation (with $\gamma_{\mathrm{L}} 13.50 \%$ compared to $11.20 \%$ of iron-crosslinked network), while showing severely diminished recovery compared to DopaK-PA/Fe(III).

\section{Conclusions}

Metal complexation has emerged as a promising cross-linking strategy for mechanical reinforcement of synthetic polymeric materials while its promise has not yet been recognized for supramolecular polymers. In the present study, we showed that mussel inspired iron coordination into supramolecular networks formed by peptides could improve mechanical properties while remaining orthogonal to the self-assembly process. We showed that enhancement of elasticity in iron cross-linked DopaK-PA/Fe(III) was one order of magnitude greater compared to physically entangled network of K-PA. We further showed pH-dependent reversibility of DopaK-PA/Fe(III) was at a comparable level to that of K-PA while covalently cross-linked DopaK-PA showed very limited reversibility after lowering $\mathrm{pH}$ to acidic levels. Likewise, recovery after high-shear load in iron coordinated peptide gel was comparable to K-PA gel. On the other hand, recovery of DopaK-PA gel was very limited, since covalent cross-linking inhibited reestablishment of the bonds. Overall, DopaK-PA/Fe(III) network showed improved mechanical properties, characteristic of chemically cross-linked networks, while retaining its original features of $\mathrm{pH}$ response and self-healing. Using a similar strategy, Dopa-mediated crosslinking can further be applied to a broad range of supramolecular systems, through which mechanical properties can be reversibly controlled. Considering underwater adhesion capacity, self-healing, and reversible bonding scheme, this work reveals important results in development of high performance hydrogels, adhesives, and coatings that can remain mechanically stable under abrasive conditions while retaining surface versatility and environmentally friendliness.

\section{Experimental Section}

Materials: All reagents used in this study were purchased from commercially available sources as analytical grade and were used as received.

Synthesis and Characterization of Peptide Amphiphiles: Fmoc solid phase peptide synthesis method was employed to manually synthesize lauryl-Val-Val-Ala-Gly-Lys-Dopa-Am (DopaK-PA), and lauryl-Val-Val-AlaGly-Lys-Am (K-PA). Rink amide MBHA resin (Novabiochem) served as the solid support. Carboxylate group activation of 2 mole equivalents of amino acid was succeeded by 1.95 mole equivalents of $N, N, N^{\prime}, N^{\prime}$ Tetramethyl-O-(1 H-benzotriazole-1-yl) uronium hexafluorophosphate (HBTU), and 3 mole equivalents of diisopropylethylamine (DIEA) for 1 mole equivalent of functional sites on the solid resin. Fmoc groups were removed at each coupling step with $20 \%$ piperidine/dimethylformamide for $20 \mathrm{~min}$. Amino acid coupling time was set to be $2 \mathrm{~h}$ at each cycle. Lauric acid served as the source of lauryl group and its coupling mechanism was similar to amino acid coupling. After synthesis, all protecting groups were removed using trifluoroacetic acid (TFA) (95\%) cleavage cocktail containing water $(2.5 \%)$ and triisopropylsilane $(2.5 \%)$. Excess TFA was removed by rotary evaporation. Peptides were then precipitated in diethyl ether overnight. The precipitate was collected and dissolved in ultra pure water. This solution was frozen at $-80^{\circ} \mathrm{C}$ followed by freeze-drying for one week. Residual TFA was removed by dissolving the whole batch in dilute $\mathrm{HCl}$ solution and freeze-drying. Small contaminants and salts were removed through dialysis using a cellulose ester dialysis membrane with molecular-weight-cut-off of 100-500 Da. After dialysis, DopaK-PA and K-PA were once more freezedried and their purity was assessed using Agilent 6530 quadrupole time of flight (Q-TOF) mass spectrometry with electrospray ionization (ESI) source equipped with reverse-phase analytical high performance liquid chromatography (HPLC). DopaK-PA and K-PA were synthesized and used with $>95 \%$ purity (Supporting Information Figure S1a,b). UV-vis spectrum of DopaK-PA at $\mathrm{pH} \approx 3$ showed that catechol side chain of Dopa remained unoxidized during the synthesis and purification steps (Supporting Information Figure S1c). Samples for analyses were prepared by dissolving freeze-dried products in ultrapure water and adjusting $\mathrm{pH}$ using sufficient amount of $\mathrm{HCl}$ or $\mathrm{NaOH}$. The $\mathrm{pH}$ of DopaK-PA solution was prepared at $\mathrm{pH} \approx 3$ and used immediately after it is dissolved in order to prevent spontaneous oxidation.

Crosslinked Gel Preparations: $\mathrm{Fe}^{3+}$ coordination to Dopa at basic $\mathrm{pH}$ was performed as previously described ${ }^{[17]}$ Unless otherwise is indicated, 20 volume units of DopaK-PA (1.25 wt\%) solution in water was mixed with 2 volume units $53.3 \mathrm{mM} \mathrm{FeCl}_{3}$ solution at $\mathrm{pH} \approx 3$. Dopa:Fe ratio in DopaK-PA/Fe(III) gels was $3: 1$. The blend was thoroughly mixed through a micro pipette. After a homogenous solution was prepared, $\mathrm{pH}$ was increased to $\approx 10$ using 3 volume units of $150 \mathrm{~mm} \mathrm{NaOH}$. Immediate color shift to wine-red was assessed as $\mathrm{Fe}(\mathrm{Dopa})_{3}$ tris-complexation (Supporting Information Figure S3 and Figure S5). ${ }^{[17]}$ 
Covalent cross-linking of DopaK-PA gel was done in the absence of iron at $\mathrm{pH}$ 10. Unless otherwise indicated, 20 volume units of $1.25 \mathrm{wt} \%$ (16 $\mathrm{mm}$ ) DopaK-PA solution in water was mixed with 2 volume units of ultra pure water at $\mathrm{pH} \approx 3$. The blend was thoroughly mixed through a micro pipette. After a homogenous solution was prepared, $\mathrm{pH}$ was increased to $\approx 10$ using 3 volume units of $150 \mathrm{~mm} \mathrm{NaOH}$. Immediate color change to pale yellow indicated formation of o-quinone (Supporting Information Figure S3 and Figure S5). Over time, color of the gel turned from pale yellow to brown (Supporting Information Figure S3), indicating formation of covalently cross-linked species of oxidized Dopa residues. ${ }^{[26]}$

Scanning Electron Microscopy (SEM): Samples for SEM imaging were prepared from $1 \mathrm{wt} \%$ gels. Following gradual exchange with ethanol, samples were dried at the critical point of carbon dioxide. A FEI Quanta 200 FEG scanning electron microscope with an ETD detector was used for visualization of peptide networks. Samples were sputter coated with $5 \mathrm{~nm}$ gold/palladium prior to imaging

Circular Dichroism (CD): CD measurements were carried out at $2 \times 10^{-4} \mathrm{M}$ peptide concentration in a 1- $\mathrm{mm}$ path length quartz cuvette. In Fe(III) cross-linked nanofibers, Dopa:Fe ratio was 3:1. A Jasco J-815 spectropolarimeter was employed with a band width of $1.0 \mathrm{~nm}$, and scanning speed of $100 \mathrm{~nm} \mathrm{m^{-1 }}$. The $190-350 \mathrm{~nm}$ spectral region was monitored for the analysis of secondary structure of peptide nanostructures.

Fourier Transform Infrared Spectroscopy (FT-IR): A Bruker VERTEX 70 was utilized to probe the secondary structure of peptide nanostructures. After $1 \mathrm{~h}$ equilibration under humid and ambient conditions, $1 \mathrm{wt} \%$ gels were instantaneously frozen in liquid nitrogen followed by freeze-drying to remove all water content. The remaining peptide network was used to form pellet with $\mathrm{KBr}$. The spectral region of $400-4000 \mathrm{~cm}^{-1}$ was scanned with 128 scan number and $4 \mathrm{~cm}^{-1}$ resolution.

Oscillatory Rheology: An Anton Paar Physica RM301 Rheometer with a $25-\mathrm{mm}$ parallel-plate configuration was used to probe the viscoelastic properties of DopaK-PA, DopaK-PA/Fe(III), and K-PA gels at pH 10. Gels were formed in situ on the lower plate of the rheometer. The final peptide concentration after gelation was set to be $1 \mathrm{wt} \%$. Shear gap distance was $500 \mu \mathrm{m}$ and total loading volume was $250 \mu \mathrm{L}$ in the measurement gap. Unless otherwise noted, all measurements were carried out at room temperature. Kinetics of gelation was probed with time-dependent rheology until the system reached a plateau, during which angular frequency $(\omega)$ and strain $(\gamma)$ were held constant at $10 \mathrm{rad} \mathrm{s}^{-1}$ and $0.01 \%$, respectively, within the linear viscoelastic range (LVR). Frequency sweep test was performed at equilibrium after $1 \mathrm{~h}$ gelation under constant strain, $0.01 \%$, with logarithmic ramping from 0.1 to $100 \mathrm{rad} \mathrm{s}^{-1}$. Amplitude sweep test was performed to determine the linear viscoelastic range of the supramolecular networks. The test was done for equilibrated samples at constant angular frequency of $10 \mathrm{rad} \mathrm{s}^{-1}$ with logarithmically ramping strain amplitude from 0.01 to $100 \%$. The $\mathrm{pH}$-dependent reversibility of self-assembled peptide networks was tested after gels were equilibrated for $1 \mathrm{~h}$ at $\mathrm{pH}$ 10. After $1 \mathrm{~h}$ sufficient amount of $\mathrm{HCl}$ was dropped onto the gels. After $10 \mathrm{~min}$ of equilibration, rheological parameters were monitored at $0.01 \%$ strain and $10 \mathrm{rad} \mathrm{s}^{-1}$ angular frequency. Thixotropic behavior was investigated as time-dependent recovery after high shear load. In the first part of the experiment, gels at equilibrium modulus were deformed in LVR, $0.01 \%$ for $3 \mathrm{~min}$. Then, strain was logarithmically ramped to $1000 \%$ within $1 \mathrm{~min}$ followed by recovery of deformation back again in the LVR, at $0.01 \%$ for 10 min. During thixotropic analyses, angular frequency was held constant at $10 \mathrm{rad} \mathrm{s}^{-1}$. Thermal properties of the gels were investigated between $20-80{ }^{\circ} \mathrm{C}$, at $10 \mathrm{rad} \mathrm{s}^{-1}$ angular frequency and $0.01 \%$ shear strain. Heating and cooling rates were set to $10{ }^{\circ} \mathrm{C} \mathrm{min}^{-1}$ with linear ramping. In order to maintain the hydration level of the gels during measurements, a solvent trap supported with a humidified environment was used during temperature-sweep tests. This system has no direct contact with the measurement system and hence does not influence the mechanical measurements. Concentration dependent analyses of the gels were presented at their equilibrium for each concentration point. Each measurement in concentration sweep was carried out under constant $10 \mathrm{rad} \mathrm{s}^{-1}$ angular frequency, and $0.01 \%$ strain.
Nanomechanical Characterizations of Mussel Inspired Peptide Nanofibers: Mechanical properties of peptide nanofibers were investigated by doublepass force-distance mapping. A detailed description of the technique has been published elsewhere and its utility was sought previously for selfassembled peptide nanofibers. ${ }^{[34,36]}$ Briefly, an atomic force microscope (AFM) (Asylum Research MFP3D) was used with built-in double-pass imaging capability. In the first pass, topography images of the air-dried nanofibers were acquired in non-contact mode. In the second pass, the cantilever dither signal is turned off, whilst the sample is vibrated at a frequency lower than the previous cantilever resonance frequency. Deflection signal was recorded using an oscilloscope, which is further processed to obtain force-distance curves. Each force-distance curve was divided into approach and retraction parts from which slope of the forcedistance curve during contact was calculated (which can then be used to infer the elastic modulus of the sample), and adhesion forces were extracted. The calibration of the measurement was done on bare silicon surface. For sample preparation, 0.05 wt\% DopaK-PA and K-PA solutions were used to form self-assembled nanofibers on one-side polished clean silicon wafer surface. Self-assembly and cross-linking reactions were done similarly to gel sample preparations.

\section{Supporting Information}

Supporting Information is available from the Wiley Online Library or from the author.

\section{Acknowledgements}

This project was supported by the Scientific and Technological Research Council of Turkey (TUBITAK) grant number 110M353, FP7 Marie Curie IRG, and COMSTECH-TWAS grant. H.C. is supported by TUBITAK-BIDEB fellowship. M.O.G. acknowledges support from the Turkish Academy of Sciences Distinguished Young Scientist Award (TUBA-GEBIP).

Received: August 13, 2012

Revised: October 10, 2012

Published online: November 1, 2012

[1] F. D. Annette, A. S. Malcolm, A. J. Middleberg, Nat. Mater. 2006, 5, 502

[2] M. de Loos, B. L. Feringa, J. H. van Esch, Eur. J. Org. Chem. 2005, 2005,3615

[3] X. Yan, D. Xu, X. Chi, J. Chen, S. Dong, X. Ding, Y. Yu, F. Huang, Adv. Mater. 2012, 24, 362.

[4] M. Ikeda, T. Tanida, T. Yoshii, I. Hamachi, Adv. Mater. 2011, 23, 2819.

[5] T. Aida, E. W. Meijer, S. I. Stupp, Science 2012, 335, 813.

[6] P. W. K. Rothemund, Nature 2006, 440, 297.

[7] W.-W. Tsai, L.-S. Li, H. Cui, H. Jiang, S. I. Stupp, Tetrahedron 2008, 64, 8504.

[8] S. Roy, A. Banerjee, Soft Matter 2011, 7, 5300

[9] N. E. Shi, H. Dong, G. Yin, Z. Xu, S. H. Li, Adv. Funct. Mater. 2007, 17, 1837.

[10] J. P. Jung, J. L. Jones, S. A. Cronier, J. H. Collier, Biomaterials 2008 29, 2143.

[11] L. Aulisa, H. Dong, J. D. Hartgerink, Biomacromolecules 2009, 10 2694.

[12] J. C. Stendahl, M. S. Rao, M. O. Guler, S. I. Stupp, Adv. Funct. Mater. 2006, 16, 499 .

[13] S. E. Paramonov, H.-W. Jun, J. D. Hartgerink, J. Am. Chem. Soc. 2006, 128, 7291.

[14] E. T. Pashuck, H. Cui, S. I. Stupp, J. Am. Chem. Soc. 2010, 132, 6041. 
[15] H. Lee, J. Rho, P. B. Messersmith, Adv. Mater. 2009, 21, 431.

[16] H. Lee, S. M. Dellatore, W. M. Miller, P. B. Messersmith, Science 2007, 318, 426.

[17] N. Holten-Andersen, M. J. Harrington, H. Birkedal, B. P. Lee, P. B. Messersmith, K. Y. C. Lee, J. H. Waite, Proc. Natl. Acad. Sci. USA 2011, 108, 2651.

[18] C. E. Brubaker, P. B. Messersmith, Langmuir 2012, 28, 2200.

[19] Q. Lin, D. Gourdon, C. Sun, N. Holten-Andersen, T. H. Anderson, J. H. Waite, J. N. Israelachvili, Proc. Natl. Acad. Sci. USA 2007, 104, 3782.

[20] H. Ceylan, A. B. Tekinay, M. O. Guler, Biomaterials 2011, 32, 8797.

[21] H. Ceylan, S. Kocabey, A. B. Tekinay, M. O. Guler, Soft Matter 2012, 8, 3929

[22] S. M. Kang, N. S. Hwang, J. Yeom, S. Y. Park, P. B. Messersmith, I. S. Choi, R. Langer, D. G. Anderson, H. Lee, Adv. Funct. Mater. 2012, 22, 2949.

[23] H. Zeng, D. S. Hwang, J. N. Israelachvili, J. H. Waite, Proc. Natl. Acad. Sci. USA 2010, 107, 12850.

[24] Z. Shafiq, J. Cui, L. Pastor-Pérez, V. San Miguel, R. A. Gropeanu, C. Serrano, A. del Campo, Angew. Chem. Int. Ed. 2012, 51, 4332.

[25] J. Monahan, J. J. Wilker, Langmuir 2004, 20, 3724.

[26] H. Xu, J. Nishida, W. Ma, H. Wu, M. Kobayashi, H. Otsuka, A. Takahara, ACS Macro Lett. 2012, 1, 457.

[27] S. W. Taylor, D. B. Chase, M. H. Emptage, M. J. Nelson, J. H. Waite, Inorg. Chem. 1996, 35, 7572.
[28] M. J. Harrington, A. Masic, N. Holten-Andersen, J. H. Waite, P. Fratzl, Science 2010, 328, 216

[29] J. D. Hartgerink, E. Beniash, S. I. Stupp, Science 2001, 294, 1684.

[30] J. D. Hartgerink, E. Beniash, S. I. Stupp, Proc. Natl. Acad. Sci. USA 2002, 99, 5133.

[31] J. D. White, J. J. Wilker, Macromolecules 2011, 44, 5085.

[32] C. Yan, D. J. Pochan, Chem. Soc. Rev. 2010, 39, 3528.

[33] N. A. Kurniawan, L. H. Wong, R. Rajagopalan, Biomacromolecules 2012, 13, 691.

[34] Y. S. Dagdas, A. Tombuloglu, A. B. Tekinay, A. Dana, M. O. Guler, Soft Matter 2011, 7, 3524.

[35] M. A. Greenfield, J. R. Hoffman, M. O. de la Cruz, S. I. Stupp, Langmuir 2009, 26, 3641.

[36] Y. S. Dagdas, M. N. Aslan, A. B. Tekinay, M. O. Guler, A. Dâna, Nanotechnology 2011, 22, 295704.

[37] G. G. Yaralioglu, F. L. Degertekin, K. B. Crozier, C. F. Quate, J. Appl. Phys. 2000, 87, 7491.

[38] G. Cinar, H. Ceylan, M. Urel, T. S. Erkal, E. D. Tekin, A. B. Tekinay, A. Dâna, M. O. Guler, Biomacromolecules 2012, 13, 3377.

[39] S. Zhang, M. A. Greenfield, A. Mata, L. C. Palmer, R. Bitton, J. R. Mantei, C. Aparicio, M. O. de la Cruz, S. I. Stupp, Nat. Mater. 2010, 9, 594

[40] J. J. Wilker, Curr. Opin. Chem. Biol. 2010, 14, 276.

[41] V. V. Papov, T. V. Diamond, K. Biemann, J. H. Waite, J. Biol. Chem. $1995,270,20183$.

[42] J. H. Waite, X. Qin, Biochemistry 2001, 40, 2887. 